\title{
Das internationale Abkommen zum Verbot von Kernwaffen - was tun?
}

https://doi.org/10.1515/sirius-2018-2007

\section{Einleitung}

Der Atomwaffenverbotsvertrag (Treaty on the Prohibition of Nuclear Weapons - TPNW) befindet sich weiterhin in der Schwebe. Er hat zwar viele Unterzeichner, aber es haben nicht genügend Staaten die Ratifikationsurkunden hinterlegt, um sein Inkrafttreten zu ermöglichen. Dementsprechend hat sich die Debatte über das Verbot von den Korridoren der Vereinten Nationen in die nationalen Hauptstädte verlagert, wo Politiker, politische Parteien und Parlamente nun entscheiden müssen, ob sie den Prozess der Ratifizierung und das Inkrafttreten des Vertrags weiter verfolgen wollen oder nicht. ${ }^{1}$ Ihre Entscheidungen stehen im Mittelpunkt der Aktivitäten der Internationalen Kampagne zur Abschaffung von Atomwaffen (ICAN), die nun alle Beteiligten unter Druck setzt. ${ }^{2}$ Was sollen die politischen Entscheidungsträger aus dem machen, was die Anhänger einer Kernwaffenfreien Welt für sie geschmiedet haben?

\footnotetext{
1 Die hier geäußerten Ansichten sind die persönlichen Ansichten des Autors und sollten keiner Institution zugeordnet werden, mit der er verbunden ist. Der Autor ist dankbar für die Kommentare zu früheren Entwürfen dieses Essays von Ivanka Barzashka, Godfried van Benthem van den Bergh, Linton Brooks, Madelyn Creedon, Lewis Dunn, Ronald Lehman, Frank Miller, Paul Schulte, Bruno Tertrais und Heather Williams. Er alleine ist verantwortlich für die hier vorgestellten Argumente.

2 Beatrice Fihn, Nobel Lecture, Oslo, 10. Dezember 2017. Dazu auch: Fihn, „Time to Step Off the Nuclear Tightrope“, The Financial Times, 7. Januar 2018.
}

\footnotetext{
*Kontakt: Dr. Brad Roberts, Direktor des Center for Global Security Research am Lawrence Livermore National Laboratory; E-Mail: roberts86@llnl.gov; eine frühere Fassung dieses Artikels erschien in englischer Sprache auf der Website des European Leadership Networks (https://www.europeanleadershipnetwork.org/policy-brief/ ban-the-bomb-or-bomb-the-ban/)
}

\section{Das Verbot so verstehen, wie es entworfen wurde}

Erstens sollten die politischen Akteure in den nationalen Hauptstädten verstehen, dass der Vertrag alleine noch kein Verbot darstellt, sondern eine Verpflichtung, irgendwann in der Zukunft ein solches Verbot zu schaffen. Angelegenheiten, die für die Wirksamkeit eines Kernwaffenverbots von zentraler Bedeutung sind - wie die Verantwortlichkeiten und Befugnisse einer „zuständigen internationalen Behörde“ für die Überprüfung und Einhaltung der Regeln -, werden ausdrücklich künftigen Verhandlungen überlassen.

Es ist kaum verwunderlich, dass die Vertragsgestalter dies auf einen späteren Zeitpunkt vertagen. Die Probleme der Wirksamkeit eines Atomwaffenverbots sind seit den frühesten Tagen des Atomzeitalters und dem Scheitern des Baruch-Plans im Jahr 1946 bekannt. Eine wirksame Lösung ist bislang nicht gefunden worden. Sie erfordert die Überwindung von zwei klar umrissenen Problemen.

- So müssen auf dem Weg vom Status quo zur globalen Null die atomar bewaffneten Staaten den Eindruck haben, dass eine glaubwürdige Garantie ihrer Sicherheit den Wert, den sie Atomwaffen zuschreiben, angemessen ersetzen kann.

- Des Weiteren müssen alle anderen Staaten davon ausgehen, dass ihre vitalen Interessen nicht durch einen Staat (oder einen nichtstaatlichen Akteur) gefährdet werden könnte, der heimlich Atomwaffen herstellt und dann einsetzt.

Für die Bewältigung des ersten Problems muss ein internationaler Mechanismus, vermutlich der Sicherheitsrat der Vereinten Nationen (VNSR), in die Lage versetzt werden, so zu handeln, dass die entwaffnenden Nationen geschützt sind - insbesondere vor Drohungen durch Atomwaffenstaaten. Bisher hat der Sicherheitsrat der Vereinten Nationen die Voraussetzungen nicht geschaffen.

Für die Bewältigung des zweiten Problems muss ein internationaler Mechanismus vorhanden sein, der zeitnah Betrug von militärischer oder anderer Signifikanz zu 
erkennen vermag und der auch Maßnahmen zur Wiederherstellung der Vertragseinhaltung ergreifen kann. Die Erfolgsbilanz des VNSR bei der Erkennung von Betrug und Wiederherstellung der Ordnung ist aber nur bestenfalls gemischt, in Bezug auf Nordkorea hat er völlig versagt. Sein Gesamterfolg hinsichtlich des Irans kann noch nicht bewertet werden, mit Blick auf den Irak ist das Ergebnis gemischt (Erfolg in Runde 1 in den 1990er-Jahren, Scheitern in Runde 2 im folgenden Jahrzehnt). Die zukünftige Wirksamkeit des VNSR in dieser Rolle scheint es erforderlich zu machen, dass die ständigen Mitglieder des Rates ihr Vetorecht aufgeben. Das wird allerdings nicht passieren. Seit dem Scheitern des ersten Abrüstungsplans 1946 sind keine weiteren bahnbrechenden Ideen $\mathrm{zu}$ diesem Thema entstanden.

Einige führende Verbotsverfechter weisen das Erfordernis von Einhaltungs- und Durchsetzungsmechanismen mit der Begründung zurück, dass eine Umgehung des Verbots nicht stattfinden würde oder nicht von Belang wäre, weil ein Betrüger keinen langfristigen Vorteil angesichts des entschiedenen Widerspruchs dieser ,zuständigen Behörde“ hätte. Vielleicht sollte man sich aber daran erinnern, dass das eine Mal in der Geschichte, an dem ein Staat ein atomares Monopol besaß, er diese Waffe auch dazu nutzte, um die bedingungslose Kapitulation eines Feindes zu erzwingen. Anschließend war diese Waffe dabei behilflich, eine Weltordnung entsprechend seiner Präferenzen herzustellen. Ein zukünftiger atomarer Monopolist müsste jedoch gar nicht den Drang verspüren, damit Kriege zu beenden oder eine auf Demokratie und Rechtsstaatlichkeit basierende UNO zu schaffen. Vielleicht geht es ihm nur um Regimesicherheit.

Zweitens sollten Politiker, die sich mit dem TPNW auseinandersetzen, sich dessen bewusst sein, dass die ICAN-Kampagne sich nicht auf alle atomar bewaffneten Staaten gleich auswirken wird. Einige werden davon unbeeindruckt bleiben, während der politische Druck in Demokratien eher Folgen haben wird. Nicht zufällig werden mit der ICAN-Kampagne progressive politische Parteien herausgegriffen, insbesondere solche, die sich derzeit in der parlamentarischen Opposition befinden. Es wird darauf spekuliert, dass diese das Kernwaffenverbot in ihre Programmatik aufnehmen und nach einem Wahlsieg zu implementieren versuchen.

So findet sich auf der Website von ICAN ein Musterbrief zur Unterstützung des TPNW, den jeder Bürger unterzeichnen und an seine Regierung senden kann. Welchen Einfluss wird eine solche Briefkampagne in Moskau, Pjöngjang oder Beijing haben? Entsprechende Bemühungen, Russland dazu zu drängen, die Verpflichtungen des INF-Vertrags wieder zu respektieren, haben in den vergangenen zehn Jahren nichts erreicht. Wenn Vladimir Putin und Kim Jong-un sich nicht dafür schämen, dass sie Menschen töten, um ihre Macht zu bewahren, werden sie sich auch kaum von diesen öffentlichen Aufrufen beeindrucken lassen. Zudem haben sie wiederholt deutlich werden lassen, wie wichtig ihnen Atomwaffen sind. Diese Waffen sind nicht nur dazu gedacht, einen atomaren Angriff der USA abzuwehren (und würden daher auch nicht verschwinden, wenn die US-amerikanischen Atomwaffen dies täten). Sie sind Werkzeuge gegen die militärische Vorherrschaft der USA und deren konventionelle Fähigkeiten, gegen deren Allianzen und gegen eine Außenpolitik, die darauf abzielt, die US-amerikanische Vision von Menschenrechten und Demokratie global voranzutreiben. Wie Präsident Putin dargelegt hat, sind Atomwaffen für Russland von entscheidender Bedeutung, um seinen Großmachtstatus, seine Souveränität und seine Integrität wiederherzustellen, und wichtige Instrumente, um die regionale und globale Ordnung in seinem Sinne zu gestalten.

Die politische Kampagne von ICAN wird also nur in demokratischen Staaten Auswirkungen zeitigen, in denen die politischen Debatten unter dem Einfluss von Nichtregierungsorganisationen stehen und wo Fragen der Moral eine große Rolle spielen. Diese Einseitigkeit wird schon heute deutlich, wenn man sich die Verteilung der ICANangegliederten NGOs anschaut. Zum Winter 2017 haben sich 468 NGOs dem Netzwerk angeschlossen. ${ }^{3}$ Weit über 200 davon befinden sich in Ländern, die mit den Vereinigten Staaten verbündet sind, während es in allen anderen atomar bewaffneten Staaten insgesamt neun ICAN-NGOPartner gibt (und keinen in Nordkorea).

Angesichts dieser asymmetrischen Ausgangslage ist nicht abzusehen, wie es durch die ICAN-Kampagne $\mathrm{zu}$ einer generellen Lockerung internationaler Spannungen oder gar zur Vertrauensbildung unter den Nationen kommen soll - Bedingungen, die im Atomwaffensperrvertrag (NPT) als unabdingbar für weitere Abrüstungsfortschritte gelten. Ein einseitiger Druck kann nur zu einer einseitigen Umsetzung führen. Demokratien sind dabei entsprechend benachteiligt. Autoritäre Staaten erhalten dadurch einen zusätzlichen Anreiz, sich ihrerseits nicht auf den TPNW einzulassen, denn der einseitige politische Druck der NGOs schwächt die Verteidigungsfähigkeit der westlichen Demokratien - mit möglicherweise gefährlichen Konsequenzen. Die Führer in Moskau, Pjöngjang und Beijing könnten in ihrem Glauben bestärkt werden, dass Demokratien schwach, leicht zu spalten und nicht willens

3 See: http://www.icanw.org/campaign/partner-organizations/. 
sind, ihre Interessen zu verteidigen, außer vielleicht unter sehr extremen Umständen.

Drittens sollten Politiker mit Blick auf das Schicksal des TPNW verstehen, dass das tatsächliche Inkrafttreten des Vertrages bei jenen Verbündeten und strategischen Alliierten der USA zu großer Verunsicherung führen wird, deren Sicherheit derzeit durch den atomaren Schirm der USA gewährleistet wird. Obwohl der Vertrag keine Klarheit darüber schafft, wie seine Einhaltung und Durchsetzung genau funktionieren sollen, ist er sehr spezifisch, was die Beendigung der Zusammenarbeit zwischen atomar bewaffneten Staaten und ihren Verbündeten betrifft. Alle Vertragsstaaten akzeptieren nämlich die Verpflichtung, ,jegliche Stationierung, Aufstellung oder den Einsatz von Atomwaffen oder anderer atomarer Sprengkörper auf ihrem Hoheitsgebiet oder an irgendeinem Ort unter ihrer Jurisdiktion oder Kontrolle zu beenden“. Außer Bündnispartnern der Vereinigten Staaten gibt es keine Länder, die auf ihrem Territorium Kernwaffen lagern oder die dafür notwendige Infrastruktur aufrechterhalten. ${ }^{4}$ ICAN-Exekutivdirektorin Beatrice Fihn hat das Verbot denn auch als eine „Gelegenheit für progressive Politiker in atomaren Gastgeberstaaten in Europa beschrieben, kühne Entscheidungen zu treffen". ${ }^{5}$ Sie hat gezielt Arten von Aktivitäten aufgeführt, die dafür infrage kommen, wie atomare Übungen, Modernisierungsprogramme und Tests von Raketen, die in der Lage wären, Kernwaffen zu transportieren. ${ }^{6}$

Der Rückzug der US-Atomwaffen würde bestimmt von vielen in Europa begrüßt werden. Aber ein Großteil der Wähler sieht das anders und ist nicht bereit, damit verbundene Sicherheitsgefährdungen in Kauf zu nehmen. Die politischen Führungskräfte der NATO-Staaten hatten in den vergangenen zwei Jahrzehnten viele Gelegenheiten, die Vereinbarungen über die atomare Teilhabe zu beenden und US-Atomwaffen aus Europa zu entfernen und haben sich jedes Mal dafür entschieden, dies nicht $\mathrm{zu}$ tun. Im Gegenteil, die erweiterte Abschreckung ist für NATO-Mitglieder (und für US-Verbündete in Nordostasien) eher wichtiger als zuvor geworden. Viele europäische Staaten sehen sich durch Russland militärisch unter Druck gesetzt, atomar und anderweitig. Manche spüren das Gewicht möglicher zukünftiger atomarer Gefahren aus dem Nahen Osten. Dementsprechend hat die NATO ihr Bekenntnis zur erweiterten nuklearen Abschreckung durch die USA regelmäßig erneuert. Mindestens drei Mal im vergangenen Jahrzehnt haben die Staats- und Regie-

4 Für mehr über dieses Thema siehe Harries 2017.

5 Fihn 2017, 47.

6 Ibid. rungschefs der NATO einstimmig eine fortgesetzte Rolle der Kernwaffen in der Abschreckungs- und Verteidigungsstrategie des Bündnisses gebilligt.

Sie verwarfen dabei die Ansicht des damaligen deutschen Außenministers Guido Westerwelle, diese Waffen seien lediglich „Relikte des Kalten Krieges““. ${ }^{7}$ Stattdessen haben die Allianzgremien ihre Einschätzung zum Ausdruck gebracht, dass diese Fähigkeiten ein notwendiger und angemessener Teil der Abschreckungsstrategie der Allianz im Sicherheitsumfeld des 21. Jahrhunderts sind. Die Präsenz US-amerikanischer Kernwaffen in Europa und die damit verbundene atomare Teilhabe hat direkte Relevanz für die Lösung der zwei wichtigsten strategischen Herausforderungen der NATO: (1) einen potenziellen Feind davon zu überzeugen, dass ein Angriff auf einen Staat tatsächlich als Angriff auf alle behandelt werden wird, und (2) einen potenziellen Feind davon zu überzeugen, dass die Vereinigten Staaten nicht einfach bei einem Krieg in Europa zuschauen werden. Die nicht-strategischen Atomwaffen der USA in Europa und die Teilhabe europäischer Alliierter bei deren Anwendung im Ernstfall sind Teil der NATO-Abschreckungsstrategie. Sie sollen demonstrieren, dass sich eine Aggression nicht lohnt. Ein Abgehen von der derzeitigen Regelung könnte dramatische negative Auswirkungen auf die europäische Sicherheit haben. Es würde als Schwächung der transatlantischen Verbindung interpretiert werden, als Unwillen der Bündnispartner, atomare Risiken für kollektive Verteidigung $\mathrm{zu}$ tragen. Sollte die NATO diesen Schritt gehen, könnte dies Präsident Putin ermutigen, zusätzliche Schritte zu unternehmen, um die von ihm abgelehnte regionale Sicherheitsordnung infrage zu stellen. Aber es könnte auch zu weiterer atomarer Proliferation in Europa führen, da einige Regierungen nach einer Aufkündigung des bisherigen Konsenses über nukleare Teilhabe und nach einem Abzug US-amerikanischer Kernwaffen aus Europa darüber nachdenken könnten, ob nicht alternative Formen der nuklearen Abschreckung für sie angemessen wären.

Darüber hinaus ist die Beibehaltung der US-Atomwaffen in Europa für die Strategie des Bündnisses für eine eventuelle Erneuerung der Rüstungskontrolle mit Russland von entscheidender Bedeutung. Wie von den NATO-Mitgliedern im Jahr 2010 vereinbart, wird in dieser Strategie ein Abkommen mit Russland zum Abbau nichtstrategischer Waffen in Europa gefordert, wobei jedoch die große Asymmetrie zwischen US-amerikani-

7 Experts support Westerwelle's quest to rid Germany of US nuclear arms, Deutsche Welle, 4. Oktober 2009; http://www.dw.com/en/ experts-support-westerwelles-quest-to-rid-germany-of-us-nucleararms/a-4753409. 
schen und russischen Waffenarsenalen zu berücksichtigen ist. Bei einem Abzug der Atomwaffen aus Europa hätte die NATO keine Verhandlungsmasse und Russland keinen Anreiz, an den Verhandlungstisch zu kommen.

Ein Abzug der nichtstrategischen Atomwaffen der USA aus Europa würde zudem eine weitere, wichtige Konsequenz haben, die bisher sehr wenig Beachtung gefunden hat. Das Ende dieser Teilhabevereinbarungen würde auch die damit verbundenen transatlantischen Konsultationen über die Regeln und Bedingungen beenden, unter denen Kernwaffen zum Einsatz kommen könnten. Die Mechanismen der Allianz zur nuklearen Mitbestimmung wurden in den 1960er-Jahren eingerichtet, um der Forderung der US-amerikanischen Verbündeten nach mehr Mitsprache über die Modalitäten des Einsatzes von Kernwaffen zu entsprechen. Die Europäer waren damals besorgt über zwei Möglichkeiten: (1) Die Vereinigten Staaten könnten ihre Kernwaffen so einsetzen, dass die Interessen der Alliierten dadurch beeinträchtigt würden; (2) sie würden ihre Kernwaffen nicht einsetzen, obwohl vitale Interessen ihrer Verbündeten gefährdet wären. Diese Ängste bleiben, wenn auch in neuer Gestalt. Würde die atomare Teilhabe der NATO enden, gäbe es keine Grundlage mehr für weitere Konsultationen über die Modalitäten des Einsatzes US-amerikanischer Kernwaffen im Wege der erweiterten Abschreckung. Der vor sechs Jahrzehnten erzielte Status der Mitbestimmung über die Modalitäten von Kernwaffeneinsätzen im Ernstfall galt damals als großer Sieg der Europäer. Sind sie jetzt bereit, ihre privilegierte Stellung wieder zu verlassen? Ist dies ein guter Zeitpunkt dafür, während in Europa die Besorgnis über schlecht kalkulierte Kriege wächst, die von Moskau, von Pjöngjang und sogar von Washington ausgehen könnten?

\section{Die Veränderung des Narrativs und die Neubelebung der Anti-Atombewegung}

Um fair zu sein: Viele der Befürworter des TPNW behaupten nicht, dass dieser allein eine Lösung für das Problem der Atomwaffen darstellt. Sie setzen sich auch nicht wirklich mit den oben genannten Mängeln auseinander. Ihr kurzfristiges Ziel besteht nicht darin, ein wirksames Vertragsregime zu schaffen. Vielmehr wollen sie erst einmal die Anti-Atom-Bewegung neu ausrichten und wiederbeleben. Dies ist für sie die Reaktion auf die ihrer Ansicht nach enttäuschende Bilanz der Atomwaffenmächte bei der nuklearen Abrüstung. Dabei geht es vor allem darum, das historische Narrativ zu ändern, wie John Borrie, einer der ICAN Aktivisten, erklärte: „Den Diskurs zu verändern - die Art und Weise, in der Dinge diskutiert werden, einschließlich der Fragen, die gestellt und beantwortet werden, muss ein Ziel für Kampagnen sein. “8 Nick Ritchie argumentierte, dass es der „Zweck des Vertrags ist [...], die Akzeptanz atomarer Gewalt zu bekämpfen und zu destabilisieren, um eine ,Legitimitätskrise' für Kernwaffen und atomare Abschreckung zu schaffen“. ${ }^{9}$ Der Zweck der Narrativveränderung besteht also darin, die öffentliche Meinung gegen Atomwaffen zu mobilisieren, was auch Beatrice Fihn bestätigt: „Der Prozess der Aushandlung eines Vertrags selbst wird die Zivilgesellschaft und öffentlichen Druck in der ganzen Welt mobilisieren. Es bietet eine konkrete Möglichkeit, die Öffentlichkeit zu mobilisieren, Medien herauszufordern und in den Parlamenten Handlung zu provozieren. Kurz gesagt, es verschafft der AntiAtomwaffen-Bewegung einen Fokus. “10

Nach dieser Denkweise wird das Ergebnis eine breite, vielleicht letztlich universelle Übereinkunft darüber sein, dass Kernwaffen wegen der barbarischen humanitären Folgen ihrer Verwendung illegitime Waffen sind. Sei man erst an diesem Punkt angekommen, sollte es einfach sein, sie abzuschaffen. So heißt es auch auf der ICAN-Website: „Waffen, die verboten sind, werden zunehmend als illegitim angesehen, verlieren ihren politischen Status und damit einhergehend auch die Ressourcen für ihre Produktion, Modernisierung und Beibehaltung. “11

Wie stellen sich Befürworter des Verbots vor, sich mit den Argumenten jener „Ausreißerstaaten“ auseinanderzusetzen, die nicht bereit sind, ihre atomare Abschreckung aufzugeben? Eine Lösung wird von vielen Vertretern der ICAN-Kampagne darin gesehen, diese Ausreißer zunächst zu umgehen. Rebecca Johnson hat zum Beispiel über die Möglichkeit der Zusammenarbeit zwischen progressiven Staaten und "charismatischen Normenunternehmern“ geschrieben, um eine Kernwaffenpolitik zu umgehen, die sie als in der Vergangenheit verwurzelt ansieht. Ebenso gilt es politische Entscheidungsträger zu umgehen, die sie für unfähig hält. ${ }^{12}$ Nick Ritchie schlägt eine weitere Richtung ein und fordert eine direkte Arbeit gegen „mächtige Interessen an Atomwaffen“ und gegen ein „realistisches

8 John Borrie, Changing the Discourse on Nuclear Weapons: What it Means for Campaigners and Why it's Important, https://www.icrc.org/ en/download/file/24543/irc97_12.pdf.

9 Ritchie 2017, 44.

10 Fihn 2017, 44-45, 47.

11 Dazu: http://www.icanw.org/why-a-ban/the-case-for-a-bantreaty.

12 Johnson 2013. 
Sicherheitsparadigma“, das er für „unerträglich“ hält.. ${ }^{13}$ John Borrie argumentiert in die gleiche Richtung, wenn er fordert, dass Aktivisten versuchen sollten, „Zweifel in den Köpfen von Entscheidungsträgern über Dinge zu säen, die sie einfach für wahr gehalten hatten. Dadurch können dann solche Leute (um-)erzogen, befähigt und für die eigene Sache eingespannt werden." ${ }^{14}$

Kurz gesagt, das TPNW ist kein ernsthafter Versuch in Richtung Atomwaffenverbot. Der Vertragstext ist vielmehr Instrument einer politischen Kampagne, deren Ziel es ist, angebliche private und institutionelle Interessen $\mathrm{zu}$ schwächen, die von der Kernwaffenabschreckung profitierten. Außerdem geht es darum, das „realistische Paradigma“ (eine der bekannten Denkschulen der Theorie der Internationalen Politik) anzugreifen und durch Mobilisierung der Öffentlichkeit einen Effekt der Umerziehung zu erzielen. Um diese Umerziehungsbemühungen voranzutreiben, stellen ihre Anhänger zahlreiche Behauptungen über Atomwaffen, ihre Rolle in der Weltpolitik und das Abrüstungsprojekt auf. Vier dieser Behauptungen verdienen hier Beachtung.

Die erste Behauptung ist, dass die atomare Abschreckung nicht funktioniert. Um die ICAN-Website zu zitieren: „Weit davon entfernt, den Frieden zu bewahren, erzeugen sie [die Atomwaffen] Angst und Misstrauen unter den Nationen. Diese ultimativen Instrumente des Terrors und der Massenvernichtung haben keinen legitimen militärischen oder strategischen Nutzen und sind nutzlos, wenn es um die heutigen realen Sicherheitsbedrohungen geht [...]. Nationen klammern sich noch immer an der fehlgeleiteten Idee der atomaren Abschreckung fest, obwohl es klar ist, dass Atomwaffen nur nationale und globale Unsicherheit verursachen.“15 John Borrie etwa schreibt: „Die atomare Abschreckung ist ein Glaubenssystem, das auf vermuteten Beziehungen zwischen bestimmten Ursachen und Wirkungen beruht [...]. Gleichwohl zeigen Studien, dass ,offizielle Experteneinschätzungen zu Fragen der Sicherheitspolitik in der Vergangenheit eine prädiktive Erfolgsrate hatten, die nur geringfügig genauer war als der Zufall." ${ }^{\text {16 }}$ Abschreckung ist dieser Ansicht zufolge nicht nur ein Glaubenssystem; es ist ein System, dass nur marginal besser als ein Münzwurf ist.

Mit der Behauptung, die atomare Abschreckung funktioniere nicht, stellen sich die ICAN-Aktivisten in der langen Diskussion darüber, ob Atomwaffen in der zweiten Hälfte des 20. Jahrhunderts zum „langen Frieden“ beige-

13 Ritchie 2017, 48-49.

14 Borrie 2017, Changing the Discourse on Nuclear Weapons.

15 Zugriff 12. Februar 2018.

16 Borrie, Changing the Discourse on Nuclear Weapons. tragen haben, auf eine Seite. Das ist im Prinzip zulässig, nur muss man sich dann auch auf die Regularien derartiger wissenschaftlich geführter Debatten einlassen - und das bedeutet auch die Auseinandersetzung mit den Argumenten der Gegenseite. Eine intellektuell rigorose Beurteilung, die 1996 durchgeführt wurde (und daher nicht durch die Notwendigkeit beeinflusst wurde, Argumente für oder gegen TPNW anzupassen), kam seinerzeit nach Abwägung aller relevanten Argumente zu dem Schluss, dass das Urteil in dieser Frage aufgrund der „Masse an widersprüchlichen Beweisen“ noch immer nicht gefällt werden könne. ${ }^{17}$ Und die ICAN-Aktivisten ignorieren eine Menge an harten Beweisen, die sogar von Kritikern der Abschreckung kommen, wonach die Existenz von Atomwaffen moderierende Auswirkungen während politisch-militärischer Krisen hatten und dass sie auch die allgemeine politisch-militärische Strategiediskussion beeinflussten in dem Sinne, dass Kriege immer undenkbarer wurden. Die Datenlage zeigt auch, dass die atomare Abschreckung nicht absolut zuverlässig ist und große Risiken birgt. Die ICAN-Aktivisten nehmen diese Debatte nicht zur Kenntnis, sondern biegen sich die komplexe und widersprüchliche Geschichte der nuklearen Abschreckung so zurecht, dass sich diese mit ihrem eigenen Narrativ und ihrer politischen Mission deckt.

Eine zweite Behauptung betrifft die Ethik der atomaren Abschreckung. Das moralische Argument gegen Kernwaffen ist, dass ihr Einsatz eine historisch einmalige humanitäre Katastrophe darstellen würde. Keiner von uns sollte diese Möglichkeit jemals vergessen. Aber genau diese katastrophalen Konsequenzen sind es, die Atomwaffen ihre starke abschreckende Wirkung verleihen. An dieser Stelle hört die Argumentation der Verbotsbewegung aber auf. Tatsächlich ist ein viel breiterer moralischer Diskurs gefordert, wollte man die Moralität der nuklearen Abschreckung beurteilen. Indem sie die Wirksamkeit der atomaren Abschreckung leugnen, weichen die Befürworter des Verbots einer der wichtigsten moralischen Fragen aus, die schon lange in der Debatte mitschwingen: ob Abschreckung im Sinne der Verhinderung von Kriegen nicht doch moralisch sein kann, selbst wenn die Waffen und die Art ihrer Anwendung (die es zu verhindern gilt) es vielleicht nicht sind. Dies ist ein Thema, über das die katholische Kirche im Laufe der Jahre immer wieder ihre Meinung geändert hat. Andere Kirchen, darunter insbesondere die orthodoxe Kirche, haben konsequent die Moral der atomaren Abschreckung unterstützt. Ein breiterer Diskurs müsste auch die moralischen Konsequenzen hinsichtlich der geretteten Leben in Kriegen berücksichti-

17 Gjelstad/Njolstad 1996. 
gen, die nur aufgrund der atomaren Abschreckung verhindert oder ohne weitere Eskalation beendet worden sind.

Ein breiterer Diskurs würde auch die Tatsache widerspiegeln, dass das humanitäre Element nicht das einzige des moralischen Diskurses über Atomwaffen ist. Diese Diskussion hat viele Strömungen. ICAN repräsentiert die Vision, „der Angst ein Ende“ zu setzen. ${ }^{18}$ Diese lebt von einer reinen, absoluten Ethik. Als er vor einem Jahrhundert über Moral und Politik schrieb, unterschied der Soziologe Max Weber aber kritisch zwischen Gesinnungsethik und Verantwortungsethik, was sich auf die gegenwärtige Debatte anwenden lässt: „Wir müssen uns klarmachen, dass alles ethisch orientierte Handeln unter zwei voneinander grundverschiedenen, unaustragbar gegensätzlichen Maximen stehen kann: es kann ,gesinnungsethisch oder ,verantwortungsethisch' orientiert sein. [...] Verantwortlich fühlt sich der Gesinnungsethiker nur dafür, dass die Flamme der reinen Gesinnung, die Flamme z. B. des Protestes gegen die Ungerechtigkeit der sozialen Ordnung, nicht erlischt. Sie stets neu anzufachen, ist der Zweck seiner, vom möglichen Erfolg her beurteilt, ganz irrationalen Taten, die nur exemplarischen Wert haben können und sollen. “19 Der Verantwortungsethiker hingegen geht davon aus, dass er für die (voraussehbaren) Folgen seines Handelns aufzukommen hat. Die vorhersehbaren Folgen eines Kernwaffenverbots sind die Schwächung der heutigen internationalen Ordnung, die auf dem Gewaltverzicht aufbaut und die dort auch in diesem Sinne funktioniert, wo die Furcht vor den katastrophalen Konsequenzen eines Kernwaffenkrieges zu einer Politik der Kriegsvermeidung und Vorsicht beiträgt. Der gesinnungsethische Diskurs des ICAN bügelt diese Überlegungen einfach ab.

Eine dritte Behauptung ist, dass das TPNW das internationale Recht stärkt. Es ist eher anzunehmen, dass genau das Gegenteil bewirkt wird. Gelegentlich schaffen Staaten internationales Recht oder Gesetzgeber Gesetze, die einfach nicht mit der Welt, wie sie ist, und mit den Interessen wichtiger Akteure übereinstimmen. Diese treffen dann Entscheidungen, die eindeutig gegen die Ziele des internationalen Vertrags oder des Gesetzes verstoßen. Man denke nur an den Kellogg-Briand-Pakt von 1928, der darauf abzielte, den Einsatz von Gewalt als Instrument der Politik zu delegitimieren. Oder an den 18. Verfassungszusatz der Vereinigten Staaten, der den Alkoholkonsum verbot (und nach breiter öffentlicher Kritik aufgehoben wurde). Dies sind möglicherweise bessere Analogien für das TPNW als das Ottawa-Verbot von Landminen. Anstatt das Völkerrecht zu stärken, kann das Verbot es auch abschwächen.

18 Fihn, Nobel lecture.

19 Weber 1992, 237.
Der vierte hier diskutierte Anspruch ist, dass die NGOGemeinschaft für die Menschen der Welt spricht. Um noch einmal Fihn zu zitieren: „Heute haben wir die Abrüstung demokratisiert und das Völkerrecht neu gestaltet [...]. Die Pflicht von ICAN ist es, diese Stimme zu sein - die Stimme der Menschheit und des humanitären Rechts, um für die Zivilbevölkerung zu sprechen [...]. Wir sind Vertreter der moralischen Mehrheit: die Milliarden von Menschen, die das Leben über den Tod wählen. “20 Auch dieser Anspruch ist kontrafaktisch, wenn man sich an die einseitige globale Verteilung der ICAN-Netzwerkmitglieder erinnert, aber auch daran, dass keinesfalls alle Menschen in Demokratien mit den Ansichten des ICAN übereinstimmen. Diese Menschen sind durchaus in der Lage, auch in solchen Angelegenheiten für sich selbst zu sprechen. Und wenn sie das tun, sind ihre Ansichten komplexer, als ICAN es behauptet. Zum Beispiel wurden Bürger von fünf NATO-Verbündeten und Israel im Jahr 2007 danach gefragt, ob sie ein durchsetzbares Verbot von Atomwaffen befürworten würden, und große Mehrheiten taten dies auch. Aber sie zeigten wenig Vertrauen in die Wirksamkeit eines multilateralen Vertragsregimes und dann werden die Bekenntnisse zur Abschaffung von Kernwaffen abhängig von Voraussetzungen, die schwer zu realisieren sein werden. In den Worten der Zusammenfassung: „Die Umfrage offenbart eine herausfordernde Ambivalenz gegenüber Atomwaffen: Es scheint, dass Menschen in Staaten, die sie [Atomwaffen] haben, denken, dass diese die Welt zwar gefährlicher machen, aber sie selbst sicherer machen." 21

Diese vierte Behauptung ist besonders beunruhigend. NGOs sind nämlich selbst ernannt, nicht gewählt. Ihre Stärke beruht auf ihrer Fähigkeit, die öffentliche Meinung zu mobilisieren und den politischen Fokus zu beeinflussen. Aber selbst wenn ihr Ziel öffentlich ist, ist ihre Identität privat. Sie werden von privaten Ressourcen unterstützt, nicht von öffentlichen. Sie sind auch niemand anderem gegenüber verantwortlich, außer ihren Geldgebern. All dies wirft eine grundsätzliche Frage nach der politischen Legitimität dieser NGOs wie ICAN auf, die sehr breite politische Ansprüche stellen. ${ }^{22}$ Gary Johns hat dazu geschrieben: „Aktivisten, die die Kontrolle durch die Öffentlichkeit umgehen können, tragen eine geringere Beweislast als der gewählte Vertreter [...]. NGOs fordern den Zugang zu den Vereinten Nationen, und das UN-Sekretariat ist sehr daran interessiert, sie zu berücksichtigen. Darüber hinaus sind nördliche NGOs eine spezielle Untergruppe der interna-

20 Fihn, Nobel acceptance speech.

21 Regeher 2007.

22 Slim 2002. 
tionalen Wählerschaft. Sie versuchen, ihre Minderheitsmeinung durch zwischenstaatliche Institutionen in eine Mehrheitsmeinung umzuwandeln. Dadurch werden Prioritäten verfälscht und Wählerschaften durch Lobbyisten ersetzt.“23

Darüber hinaus haben die Menschen bereits politische Vertreter - zumindest diejenigen, die in Demokratien leben. Und ihre gewählten Vertreter tragen moralische und politische Verantwortung, die NGOs nicht haben. Indem sie den Amtseid leisten, sind sie aufgerufen, nach Webers „Verantwortungsethik“ zu handeln. In diesem Zusammenhang sei nur an den warnenden Hinweis erinnert, den US-Präsident Barack Obama in seiner Nobel-Dankesrede geäußert hat: „Als Staatsoberhaupt, das geschworen hat, meine Nation $\mathrm{zu}$ beschützen und $\mathrm{zu}$ verteidigen, kann ich mich nicht allein an ihren Beispielen orientieren [in Bezug auf Mahatma Gandhi und Martin Luther King und ihr Engagement für Gewaltlosigkeit] [, ...] ich stelle mich der Welt, so wie sie ist, und kann angesichts der Gefahren für das amerikanische Volk nicht tatenlos zusehen." 24

So ehrenwert sie auch sein mögen, die Inhaber von NGO-Ämtern leisten keinen Eid, um die Bevölkerung und die Verfassung eines Staates zu schützen. Sie können sich den Luxus leisten, ihre Weltanschauung völlig eindimensional zu verfolgen, während gewählte Politiker versuchen müssen, mit der Welt in all ihrer Komplexität umzugehen und ihre Rolle beim Schutz derer zu erfüllen, die sie vertreten. Diese Gruppe darf Abschreckungsargumente nicht so einfach beiseitelegen.

\section{Empfehlungen: Stillstand zum Vorteil machen}

Der Verbotsvertrag verdient nicht die politische Unterstützung, die seine Mitgliedschaft erweitern und sein Inkrafttreten ermöglichen würde. Er sollte in der Schwebe bleiben - als Protestmedium für diejenigen, die nichts zu verlieren haben. Selbst Länder ohne Atomwaffen, insbesondere solche, die im Bündnis mit Atomwaffenstaaten stehen, sollten sich vorsehen, die internationale Kampagne zu unterstützen. Sie sollten den Schaden berücksichtigen, den das Verbot für die Sicherheit gefährdeter Staaten und für die internationale Ordnung im Allgemeinen bedeuten könnte.

23 Johns 2004, 56.

24 President Barack H. Obama, A Just and Lasting Peace, Nobel Lecture, Oslo, Norway, 10. Dezember 2009.
Vielmehr ist ein substanzieller Dialog über atomare Abschreckung und Abrüstung nötig, der nicht darauf abzielt, Druck auszuüben und politische Akteure zu beschämen oder umzuerziehen. Ein solcher Dialog müsste das Nachdenken darüber anregen, welche Bedingungen geschaffen werden müssen, um realen Fortschritt in Richtung auf das endgültige Ziel der Abschaffung von Kernwaffen zu ermöglichen. Dieser Weg ist mit harten Problemen gespickt, aber Klarheit darüber zu gewinnen ist unerlässlich, um die Versuchung vorschneller Lösungen zu überwinden. Eine aufgeklärte Debatte zu dieser Frage könnte dazu beitragen, einen sicherheitspolitischen Konsens zu erneuern.

Ein solcher Dialog müsste das Nachdenken über die tatsächliche Rolle von Atomwaffen für die Sicherheit unserer Gesellschaften anregen. In den meisten westlichen Ländern ist das Denken über nukleare Abschreckung aber noch tabuisiert. Dieses Tabu hat etwas mit der Stigmatisierung zu tun, die von den Verbotsaktivisten seit längerer Zeit betrieben wird. Man muss aber auch den mangelnden Mut führender westlicher Politiker nennen, die einerseits aus Gründen der Verantwortungsethik nicht bereit sind, die atomare Abschreckung aufzugeben, die sich aber andererseits der Unbeliebtheit von Atomwaffen bewusst sind und daher nicht die offene Debatte wagen. Das ist für den notwendigen öffentlichen Diskurs toxisch.

Ein solcher Dialog könnte auch neue Interessengemeinschaften sowohl in der staatlichen als auch in der nichtstaatlichen Welt schaffen. Gerade viele junge Leute sind heute unbeeindruckt von den erbitterten Kämpfen zwischen den Rüstungsgegnern und den Abschreckungsbefürwortern. Ein Zustrom von offenen Köpfen und pragmatischen Geistern würde da guttun. Wenn sich dabei TPNW und ICAN unwissentlich als hilfreich erweisen sollten, das Narrativ auf diese ungewollte Weise zu verändern, sollten wir ihnen dankbar sein.

Was sollten unsere gewählten Politiker tun, wenn sie eine Entscheidung darüber treffen müssen, wie sie mit einer Ratifizierung und dem Inkrafttreten des Verbots umgehen sollen? Sie sollten beides ablehnen. Ein Votum für das Verbot mag für viele eine lockere Anti-AtomkraftProtestabstimmung sein, aber seine tatsächlichen Auswirkungen könnten weitreichend und gefährlich sein. Das Verbot bietet keine tragfähige Lösung für das atomare Problem. Das Verbot wird wahrscheinlich in seinen Auswirkungen einseitig sein und die westlichen Demokratien massiv benachteiligen. Die erweiterte Abschreckung würde in Gefahr geraten, die Welt kriegerischer werden. Aus dem pazifistischen Protest könnte am Ende Schaden resultieren. 
Vor allem sollten unsere gewählten Politiker sich überlegen, welche Rolle sie in dieser Angelegenheit spielen wollen. Es ist zu hoffen, dass mit der Schönfärberei aufgehört, leichtfertige und moralisierende Rhetorik zurückgenommen und eine breite, pragmatische, aber prinzipienorientierte Untersuchung der vor uns liegenden Herausforderungen begonnen wird. Ziel muss es sein, eine praktische Agenda für die atomare Risikoreduktion und atomare Ordnung in den kommenden Jahren zu führen. Sie könnte damit beginnen, indem man die schwierigen Fragen stellt.

\section{Literatur}

Fihn, Beatrice (2017): The Logic of Banning Nuclear Weapons, Survival, 59 (1) 43-50.

Gjelstad, Jorn/Njolstad, Olav, Hrsg. (1996): Nuclear Rivalry and International Order. Oslo: PRIO.

Harries, Matthew (2017): The ban treaty and the future of U.S. extended nuclear deterrence arrangements, in: Shetty, Shatabhisha/Raynova, Denitsa (Hrsg.): Breakthrough or
Breakpoint? Global Perspectives on the Nuclear Ban Treaty. London: European Leadership Network, 50-57.

Johns, Gary (2004): Relations with Nongovernmental Organizations: Lessons for the UN, Seton Hall Journal of Diplomacy and International Relations, 5 (2), 56.

Johnson, Rebecca (2013): Arms Control and Disarmament Diplomacy, in Cooper, Andrew F./Heine, Jorge/Thakur, Ramesh, Hrsg.: The Oxford Handbook of Modern Diplomacy. Oxford; Oxford University Press, 453-465.

Regeher, Ernie (2007): Nuclear disarmament or nuclear ambivalence? Survey conducted by the Simons Foundation and the Angus Reid Strategies Corporation, New York: Simons Foundation, http://ploughshares.ca/wp-content/ uploads/2007/10/brf074.pdf.

Ritchie, Nick (2017): The Treaty on the Prohibition of Nuclear Weapons: delegitimizing unacceptable weapons, in: Shetty, Shatabhisha/Raynova, Denitsa (Hrsg.): Breakthrough or Breakpoint? Global Perspectives on the Nuclear Ban Treaty. London: European Leadership Network. 44-49.

Slim, Hugo (2002): By What Authority? The Legitimacy and Accountability of Non-governmental Organizations, Paper prepared for the International Council on Human Rights Policy, January $10-12$.

Weber, Max (1992): Politik als Beruf, in: Mommsen, Wolfgang J./ Schluchter, Wolfgang (Hrsg.): Max Weber Gesamtausgabe. Abt. I, Band 17. Tübingen; Mohr Siebeck, 157-252. 